\title{
Modelling user situations with smartphones
}

\author{
Seung-Ik LEE, YunKyung PARK \\ Electronics and Telecommunications Research Institute, Korea \\ the_silee@etri.re.kr, parkyk@etri.re.kr
}

\begin{abstract}
This paper presents a preliminary study on modelling personal experiences, centered on the design and the issues of the modelling. The proposed personal experience gathering framework aims at providing a ground for circulating and utilizing personal mobile contents by giving them semantic meaning through automatic annotation. Among various kinds of personal experiences, the framework models spatio-temporal information, social relations, personal interests, and multimediacontent-related activities. This paper defines the data needed, explains the ways they are (pre-) processed and annotated automatically.
\end{abstract}

Keywords - Personal experience, knowledge store, mobile, knowledge, platform, collection

\section{INTRODUCTION}

As diverse smart devices and advanced mobile Internet environment evolve, there has been a so-called mobile service Big Bang including M2M (Machine to Machine) communications, SNS (Social Network Service), and App stores.

The mobile services market features globalization, collective intelligence formed by market opening, and exclusive possession by pre-emption. Especially, most of the profits are being created in the service platforms. In such environments, the business is being transited from the provider oriented one-way services, such as the closed services oriented by existing carriers and high-value oriented premium services, to user-centered platforms that support the production of their contents.

The paradigm is changing into the direction that mobile users are no longer one-sided consumers but also producers of the information and services. In this mobile environment, a variety of information and content are created and cumulative, and the linkage between social networks from big data and the knowledge and thinking more is becoming more important.

In this environment, to prevent the user's digital assets and public information, a knowledge and information-based mobile knowledge service platform is necessary such that the assets are open and utilized creatively based on appropriate rights.

The platform (see Figure 1) are expected to provide 1) the real-time collection/annotation/management, 2) the generation of new knowledge tailed to user's needs and circumstances by fusing the user experience and the experts' knowledge, and 3) an environment where the inconvenience to use that occurs because of the inherent limitations of mobile devices is overcome and users can easily and comfortably use knowledge services and develop related applications.

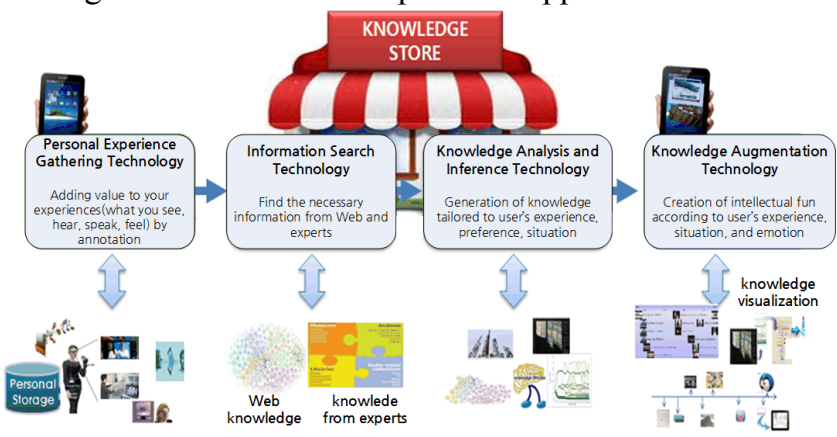

Figure 1. Mobile knowledge service platform

In this paper, we propose a user experience collection and management framework that gathers, automatically annotates, and manages personal experiences. The framework facilitates knowledge services based on personal experiences.

\section{RELATED WORK}

Microsoft performed the MyLifeBits [1] project to develop a technology for collection of digital information (radio, TV, phone call, e-mail, search, pictures, etc.) related to an individual in order to construct a complete personal history by digitizing personal experiences in daily life. This project focused on automated techniques to facilitate building database with the annotations related to the digital information made by hand.

In Europe, a project called Memories for Life was performed to lead to a more effective use and management of both the human and computerised memory. It aims to produce an understanding of what is common in memory systems and use that knowledge to improve efficiency, recall and information management across human, personal, social and work domains [2].

Yahoo has been providing the Life Map service [3]. The service displays the photos taken at the places visited by the user on the map. Also, you can conveniently browse and share travel memories conveniently with your family or friends. In addition, travel information such as attractions, accommodations, restaurants can be shared with your friends as well as the general public.

Kiyoharu Aizawa, Professor in the University of Tokyo, Japan led the "Life Log for Ubiquitous Environment" project where wearable devices were utilized to automatically log 
personal everyday life information to save the individual's experiences easily and efficiently search and manage them [4].

J. Wen et al. [5] proposed the Cyber-I (Individual) concept corresponding yourself in cyber space. Cyber-I represents an individual in cyber space with characteristics that requires immediate update such as emotional or physical condition and with characteristics that requires gradual additions or changes such as social relationships, past experiences, physical properties (gender, face, hair color, fingerprints, pupil, etc.), or personal characteristics (habits, hobbies, interests, religion).

However, those studies have a few limitations. First of all, much of the studies focused on simply collecting raw data and managing them. In other words, the collected information has no additional annotations such that it is difficult to use the information from actual services. Second, it lacks a kind of realism. It is undesirable to expect that users will put on specially designed, not commercially available, not portable devices in everyday life. Third, the cumulative personal information will be lost due to the limits of being annotated with semantic meaning as knowledge. Fourth, the collected information is one-dimensional. Much of the existing studies utilize one or two kinds of information, for example, photo, location, and time, video footage or digital information. However, in order to grasp an individual's daily life as exactly as possible, we argue that it is necessary to take every possible information into consideration including those aforementioned as well as others related to, for example, social, temporal, spatial context.

\section{Personal Experience Collection}

\section{A. Design Principles and Assumptions}

1) Multidimensional Personal Experience Model: The exact meaning of the collected information on individual's daily life has to be clearly identified. According to Kofod-Petersen and Cassens [6], the factors that govern the situation of an individual are composed of personal, task, spatio-temporal, environmental, social contexts. Personal context refers to the information about the individual; task context refers to the activities the individual performs; environmental context refers to the tools or means with which the individual perform activities; and social context means relationships with others.

In this study, we adopt the findings of Kofod-Petersen and Cassens [6] and propose a personal information model composed of spatio-temporal, social, Events, and personal profiles.

TABLE 1. PERSONAL EXPERIENCE MODEL

\begin{tabular}{|l|l|l|}
\hline \multicolumn{1}{|c|}{ Profile } & \multicolumn{1}{|c|}{ Contents } & $\begin{array}{l}\text { Relation to Kofod- } \\
\text { Petersen and } \\
\text { Cassens' taxonomy }\end{array}$ \\
\hline $\begin{array}{l}\text { personal } \\
\text { profile }\end{array}$ & name, sex, email, etc. & personal context \\
\hline $\begin{array}{l}\text { social } \\
\text { profile }\end{array}$ & social relation & social context \\
\hline $\begin{array}{l}\text { interests } \\
\text { profile }\end{array}$ & $\begin{array}{l}\text { user's current interests. } \\
\text { For example, fishing, } \\
\text { driving, golf, etc. }\end{array}$ & personal context \\
\hline
\end{tabular}

\begin{tabular}{|l|l|l|}
\hline $\begin{array}{l}\text { spatial- } \\
\text { temporal } \\
\text { profile }\end{array}$ & $\begin{array}{l}\text { log of user's visiting } \\
\text { places at a specific } \\
\text { time; user's activities at } \\
\text { a place; and the modes } \\
\text { of transportation to a } \\
\text { place }\end{array}$ & $\begin{array}{l}\text { spatial-temporal } \\
\text { context, } \\
\text { task context, } \\
\text { environmental } \\
\text { context }\end{array}$ \\
\hline event profile & $\begin{array}{l}\text { multimedia contents } \\
\text { creation/play log, phone } \\
\text { call log, sms log, credit } \\
\text { card log }\end{array}$ & $\begin{array}{l}\text { task context, } \\
\text { environmental } \\
\text { context }\end{array}$ \\
\hline
\end{tabular}

Personal profile includes basic information about the individual. Basic information includes, for example, name, gender, age, phone number, email, contacts, and more. Social profile models the relationship between individuals. Interests profile models personal interests, for example, skiing, golf, economy, history, and so on. Spatio-temporal profile models when/where/what activity an individual did; For example, an individual was working on a weekday at his office or went to home from work by car. Event profile models what happened to an individual or event-actions the individual did at a specific time. Note that an activity in spatial-temporal profile has duration such that there is a gap between the start and end time of the activity while event-actions conceptually has no duration. For example, working at office or housekeeping is an activity and taking a photo or listening to music is an event-action. Note, however, although the act of listening to music actually has some duration, what's modelled is the moment when the individual pressed the button to play music. Therefore, listening to music is conceptually modelled as event-action.

2) Automatic Annotation: Without appropriate annotations, the collected information cannot be used for services that need some semantic meaning from the information. However, requesting users to manually annotate its semantic meanings on the collected data has many difficulties. For example, sometimes users forget to annotate, sometimes they give wrong annotations, and sometimes they feel annoyed, and so on. Therefore, it is desirable to automatically annotate the collected information without any user intervention.

3) Utilizing Smartphones: To collect an individual's daily life, the device has to be portable, commercially available, and everyday life-capable. Fortunately, recent smart phones have various kinds of sensors such as GPS, accelerometer, light, proximity, gravity, orientation, and temperature, which can be used for collection raw data for daily life log.

Much of the existing research utilizes specially designed attachments to record the individual's daily life. However, considering the availability and utilization of such devices, it is not easy in real life for individuals to wear such equipment.

Therefore, when an individual collects his experience and information, it's best to use a smartphone equipped with various sensors. We can gather various sensory information from the equipped sensors in smartphones and in addition take advantage of the secondary, non-sensory information (for example, to take advantage of the Internet) to record the daily life of individuals. 


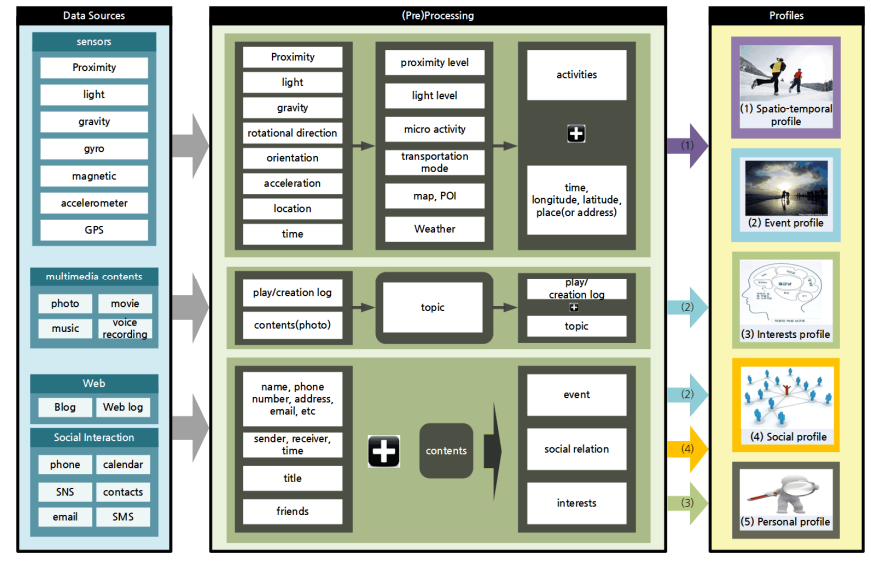

Figure 2. Design diagram of the personal experience data model

\section{B. Design}

1) Spatio-Temporal Profile: In this profile, an individual's spatial and temporal information are recorded. This means that when/where/what an individual did. Based on the transportation trajectories of an individual from GPS data, semantic trajectories are extracted, and then the places are determined utilizing other information necessary. A place is described with its name and its category.

Typically, location-based systems or GIS systems use user's time and location (latitude and longitude). However, when you want to provide higher-level services, it is necessary to know the semantic meaning of the location. Semantic meaning of a location (latitude $=x x x . y y y$, longitude $=\mathrm{mmm} . \mathrm{zzz}$ ), is that the location is, for example, McDonalds and its category is fast-food. Therefore, this study aims at providing this semantic meaning to a location automatically, i.e., without any user intervention.

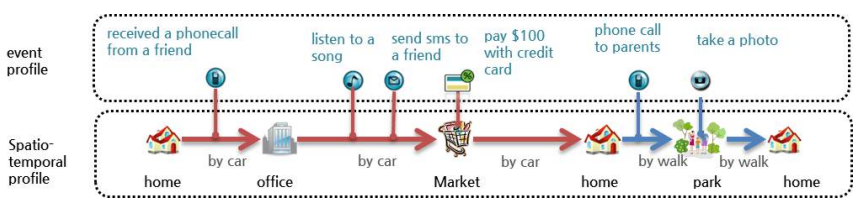

Figure 3. Spatio-temporal profile augmented with events

Figure 3 shows an example of a spatial-temporal profile augmented with events from event profile. As you can see, the spatial-temporal profile is basically a series of 'move' and 'stay', i.e., semantic trajectory [7]. A stay means the user stays there for more than a period of time. A stay is composed of intime, out-time, a coordinate, place name, place category, and main activity. A move from a place to other place is composed of abstracted path (for example, a start point and an end point), travel time, and transportation mode.

2) Event Profile: This profile record the events occurred to a user or the user's event actions. An event action is for example, sending SMS and receiving a phone call. As shown in Figure 3, multimedia play or creation (in case of a photo), sms, phone calls, credit card payment, and the like are logged with the time when the events occur. Therefore, combining spatial-temporal with event profiles gives an augmented user behavioural record.
3) Interests Profile: Interests profile record what kinds of subject the user is interested in. For this purpose, we can utilize the user's blog, weblog, sns, sms, and emails. The contents of these data sources are analysed by text mining techniques to extract a limited number of interests.

4) Social Profile: Social profiles are for modelling user's social relationship. The data sources for the profile are phone logs, sms, contacts, history, calendar, sns, email, etc. Unlike the interests profile where the contents of the data sources are analysed, social profile uses metadata only. For example, in the case of phone calls, send/receive, correspondent, time, and duration are used. Using these metadata, the existence, type, intensity, direction, and so on, are analysed.

5) Personal Profile: It models user's static information. It includes, for example, gender, age, phone number, e-mail, and the like that are not frequently changed. Therefore it is reasonable to ask the user to input them directly.

\section{Issues}

With the design aforementioned, in order to acquire user's experiences, the following parts should be decided at implementation time.

1) Supporting Devices: One of the first things that should be decided is the supporting smartphones where the personal experience collection framework will be performed. This is because the types of sensors equipped with smartphones, operating systems and event their versions are diverse depending on the smartphones. You should also take it into consideration that developing an app in the Android operating system is, in general, known to give more degrees of freedom.

2) Frequency of Data Acquisition: As smartphones have a wide variety of sensors attached to them, the frequencies of collecting the sensory information should be carefully decided according to the types of the sensors and the purpose. For example, if you want to extract the user's micro activity (i.e., walk, stand, sit ...), the frequency may be several times a second while others may be every few seconds. In addition, weather information obtained from Web not from a smartphone sensor, may be collected for example, every hour.

Therefore, the properties of the data to be collected and their purpose should be carefully considered to decide the appropriate acquisition cycle. Too frequent collection than is necessary spends the smartphone's battery too much, causing the user feel reluctant to collect data.

3) Places: To extract the place name and the category from locational coordinates, the first thing we need to consider is to user map and POI (Points of Interests) information. This information can be obtained from website such as Google or other portal webs. For example, Google's public APIs allows us to use the GPS coordinates as input to get the nearby POIs. However, this does not mean that the user is at a specific place. The returned list is just candidates around the user and you should decide which one is the real place the user currently is. What is worse, the list may not have the correct answer. That is, the user may be in none of the returned candidate places.

Therefore, it is almost impossible to exactly pinpoint the place and its category with using only GPS, map, and POI information. We need to contrive a new method to exactly 
extract the place name and the category. This still remains undecided in our study.

4) Transportation Mode: In the spatial-temporal profile, the transportation mode (by walk, car, train, ship, airplane, bus, etc.) between two visited places should be logged. As we want it to be automatically annotated, we should use the smartphone's sensors to infer the transportation model. Fortunately, many previous studies have shown promising results on inferring transportation modes [8][9]. This study will borrow some ideas from the results of the previous studies.

5) Separating Semantic Trajectories: A user's transportation is a sequence of stops (at places) and moves (transportation). From this sequence, we need to somehow separate one trajectory from others.

There can be a variety of criteria, for example stay time and the types of places. For example, if a user stays at a place more than a certain amount of time, the place can be the last place of its enclosing trajectory. Or if a user stays at a specific type of place, for example, home or office, then the place can be the last of its enclosing trajectory.

Typically, as users leave their home and return to home in a normal daily life, it is reasonable to decide home as a beginning and terminating place in a trajectory.

6) Interests List: It is regarded as almost impossible to extract a user's interests thoroughly through text mining without any limitations. Thus, certain constraints must be determined by placing a limited number of interests such that we can deploy text mining techniques to extract them.

7) Data Storing Methods: As the aforementioned profiles are eventually to be used by other services, appropriate storage format and the ways they are stored need to be decided. This may depend on the kinds of services that will use these profiles. Candidates are simple text files, XML, or database such SQLite [10]. If a wide variety of queries are expected, databases seem to be a good solution.

\section{IV.CONCLUSION}

In this paper, we have proposed a framework for collecting user experiences. This framework collects spatial-temporal information, social relationships, interests, personal information, and etc. This paper has defined the necessary sensors, sensor (pre) processing, and finally, the detailed information logged on the profiles. In addition, a number of issues to be solved have been described to help implement the framework.

As further research, the aforementioned issues need to be analysed, decided, and applied to the actual implementation.

\section{ACKNOWLEDGMENT}

This research was supported by the $\mathrm{KCC}($ Korea Communications Commission), Korea, under the R\&D program supervised by the $\mathrm{KCA}$ (Korea Communications Agency)"(KCA-2013- 12-911-04-005)

\section{REFERENCES}

[1] MyLifeBits website. Available: http://research.microsoft.com/enus/projects/mylifebits/ http://www.memoriesforlife.org/index.

[3] Yahoo Life map website. http://kr.iy.gugi.yahoo.com/lifemap/lifemap.html

[4] D. Tancharoen, T. Yamasaki, K. Aizawa, "Life log platform for continuous and discrete recording and retrieval of personal media," International Workshop on Advanced Image Technology (IWAIT), pp. 207-212, 2007.

[5] J. Wen, K. Ming, F. Wang, B. Huang, J. Ma, "Cyber-I: Vision of the Individual's Counterpart on Cyberspace," IEEE International Conference on Dependable, Autonomic and Secure Computing, pp. 295-302, 2009

[6] A. Kofod-Petersen, J. Cassens, "Using activity theory to model context awareness," Lecture Notes in Computer Science. vol. 3946, pp. 1-17, 2006.

[7] Z. Yan, D. Chakraborty, C. Parent, S. Spaccapietra, K. Aberer, "Semantic trajectories: mobility data computation and annotation," ACM Transactions on Intelligent Systems and Technology, vol. 9, no. 4, article 39, 2012.

[8] S. Wang, C. Chen, J. Ma, "Accelerometer based transportation mode recognition on mobile phones," Proceeding of the 2010 Asia-Pacific Conference on Wearable Computing Systems, pp. 44-46, 2010.

[9] S. Reddy, M. Mun, J. Burke, D. Estrin, M. Hansen, and M. Srivastava, "Using mobile phones to determine transportation modes," ACM Transactions on Sensor Networks, vol. 6, no. 2, article 13, 2010.

[10] SQLite website. Available: http://www.sqlite.org/

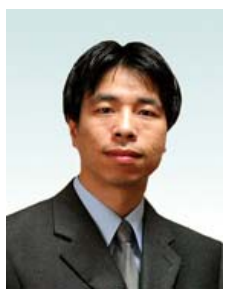

Seung-Ik Lee received his $\mathrm{MS}$ and $\mathrm{PhD}$ in computer science from Yonsei University, Seoul, Korea, in 1997 and 2001, respectively. $\mathrm{He}$ is currently working for ETRI(Electronics and Telecommunications Research Institute), Korea. His research interests include evolutionary computation, intelligent robot control, mobile situation modeling, and knowledge representation and modelling.

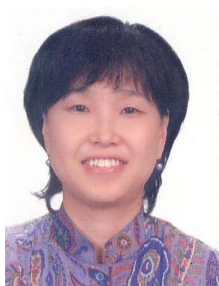

YunKyung Park received her MS in computer science from ChungNam University, Daejeon. She is currently working for ETRI(Electronics and Telecommunications Research Institute), Korea. Her research interests include knowledge representation and processing, mobile services. 